\title{
Polypharmacy or medication washout: an old tool revisited
}

This article was published in the following Dove Press journal:

Neuropsychiatric Disease and Treatment

19 October 2011

Number of times this article has been viewed

\section{Daniel A Hoffman' \\ Mark Schiller ${ }^{2}$ \\ James M Greenblatt ${ }^{3}$ \\ Dan V losifescu ${ }^{4}$}

'Neuro-Therapy Clinic Inc, Denver, $\mathrm{CO} ;{ }^{2}$ Mind Therapy Clinic,

2299 Post Street, Suite I04A,

San Francisco, CA; ${ }^{3}$ Comprehensive

Psychiatric Resources, Waltham, MA;

${ }^{4}$ Mood and Anxiety Disorders

Program, Mount Sinai School of

Medicine, New York, NY, USA

Correspondence: Daniel A Hoffman

7800 E Orchard Rd, Suite 340,

Greenwood Village, CO 8011 I, USA

Tel +l 303 74I 4800

$\mathrm{Fax}+|30374| 2244$

Email daniel@hoffmanemail.com
Abstract: There has been a rapid increase in the use of polypharmacy in psychiatry possibly due to the introduction of newer drugs, greater availability of these newer drugs, excessive confidence in clinical trial results, widespread prescribing of psychotropic medications by primary care, and pressure to augment with additional medications for unresolved side effects or greater efficacy. Even the new generation of medications may not hold significant advantages over older drugs. In fact, there may be additional safety risks with polypharmacy being so widespread. Washout, as a clinical tool, is rarely done in medication management today. Studies have shown that augmenting therapy with additional medications resulted in $9.1 \%-34.1 \%$ dropouts due to intolerance of the augmentation, whereas studies of medication washout demonstrated only $5.9 \%-7.8 \%$ intolerance to the washout procedure. These perils justify reconsideration of medication washout before deciding on augmentation. There are unwarranted fears and resistance in the medical community toward medication washout, especially at the moment a physician is trying to decide whether to washout or add more medications to the treatment regimen. However, medication washout provides unique benefits to the physician: it establishes a new baseline of the disorder, helps identify medication efficacy from their adverse effects, and provides clarity of diagnosis and potential reduction of drug treatments, drug interactions, and costs. It may also reduce overall adverse events, not to mention a potential to reduce liability. After washout, physicians may be able to select the appropriate polypharmacy more effectively and safely, if necessary. Washout, while not for every patient, may be an effective tool for physicians who need to decide on whether to add potentially risky polypharmacy for a given patient. The risks of washout may, in some cases, be lower and the benefits may be clearly helpful for diagnosis, understanding medication effects, the doctor/patient relationship, and safer use of polypharmacy if indicated.

Keywords: polypharmacy, medication washout, risk of polypharmacy

\section{Introduction}

"Polypharmacy" refers to the concurrent use of multiple medications in a single patient. However, the use of the term polypharmacy in the published literature is somewhat imprecise and has different meanings according to different authors. Most often, the definition of polypharmacy is made with regard to the specific number of medications prescribed. Thus, polypharmacy is alternatively defined as the use of two or more psychiatric medications in the same patient, ${ }^{1}$ or as the use of two or more medications to treat the same condition, use of two or more drugs of the same chemical class, or use of two or more drugs with the same or similar pharmacologic actions, ${ }^{2}$ or as prescribing of three or more medications for the same indication, or prescribing of two or more medications with the same or similar mechanisms of action(s) used for the same indication. ${ }^{3}$ 
However, using an absolute numeric threshold to define polypharmacy is inherently imprecise, because the number of medications considered optimal does vary for different psychiatric disorders. For example, optimal treatment in schizophrenia or in major depressive disorder is thought to involve most often one and generally no more than two medications - a practice reflected in the design of the large National Institutes of Health studies, ie, Clinical Antipsychotic Trials in Intervention Effectiveness (CATIE) and Sequenced Treatment Alternatives to Relieve Depression $\left(\mathrm{STAR}^{*} \mathrm{D}\right) .{ }^{4,5}$ In contrast, nearly half (48\%) of the patients with bipolar disorder treated in the best practice pathway of the National Institute of Mental Health Systematic Treatment Enhancement Program for Bipolar Disorder (STEP-BD) study received treatment with three or more medications. ${ }^{6}$

Another factor adding to the imprecision of the definition is that while use of multiple medications can have negative consequences, it can sometimes be an effective clinical intervention. Therefore, any meaningful discussion of polypharmacy must make the distinction between rational and irrational polypharmacy (which are also discussed later in this paper); the former representing an attempt to match very unusual and severe clinical presentations with pharmacological combinations having proven synergistic effect, while the latter describe haphazard combinations of medications with no distinguishable clinical or pharmacological rationale. With these caveats in mind, we will discuss polypharmacy as the practice of prescribing more than two medications in a single patient for a single indication, or prescribing of two or more medications with the same or similar mechanisms of action. We will particularly focus on the irrational aspects of polypharmacy and their negative consequences for patients. We have identified pertinent literature for this review with a computer-aided search of the US National Library of Medicine PubMed database for articles published between 1980 and 2011 in English. The key words used for the search were "polypharmacy", "antidepressant augmentation", "antipsychotic polypharmacy", "polypharmacy in psychiatry and risk", "trial and error medications" and "medication washout". The reference lists of reports identified were used to find additional publications.

There has been a rapid increase in the use of polypharmacy in psychiatry. The reasons may be multifactorial, such as an increasing number of available medications targeting new and different symptoms and receptors, or even the pressure on psychiatrists to focus on medication treatment. Regardless of the reasons, the trend is clear, with psychiatrists now frequently seeing patients presenting on multiple psychiatric medications.

Recent research raises doubts about the degree to which psychopharmacological treatment has kept pace with our advances in understanding the brain and psychiatric disorders. There is a plethora of new psychiatric medications, but there is growing recognition that these new generation medications may not hold significant advantages over older medications despite their higher costs. In 2006, DePaulo ${ }^{7}$ observed that, when viewed together, the three major studies (ie, STAR*D, CATIE, and STEP-BD) question whether or not modern pharmacological treatments are better than older interventions, notwithstanding additional costs. While none of these studies (STAR*D, CATIE, and STEP-BD) have focused primarily on comparing older and newer treatments, such contrasts ${ }^{8-11}$ do not suggest any dramatic advantages for the newer medications.

In 2009, Insel ${ }^{12}$ made similar observations to those of DePaulo, noting that second-generation medications have consistently demonstrated no significant advantage compared with first-generation medications in multiple comparative effectiveness studies funded by the National Institute of Mental Health. He also felt that current medication regimes help too few people improve from the perspective of side effects, and a recent prospective study has now even called into question the widely held belief that second-generation antipsychotics produce a lower incidence of tardive dyskinesia. ${ }^{13}$

While newer psychotropic medications might have some advantages over older ones, we may be using them incorrectly. Clearly, there are inadequacies in our current diagnostic nomenclature based as it is on descriptive psychiatry. More than likely, our diagnoses encompass several biological syndromes, which may require different pharmacological treatment. The current state of basing therapies on these descriptive diagnostic categories is insufficient. In 2011, Frances ${ }^{14}$ suggested that this approach may be limiting. In the same article, Insel ${ }^{14}$ also mentioned that the National Institute of Mental Health is reallocating resources to reclassify psychiatric diagnoses by 2020, and later said they would be reformulated under the umbrella of Brain Disorders. Given the significant changes taking place, a major concern for many psychiatrists is how to select the most effective treatment today, and over the next decade, while we transition into an evidence-based neuroscience. ${ }^{14}$

This paper centers on the risks and benefits of increasingly prevalent polypharmacy and examines the risks and benefits of the underutilized tool of medication washout for appropriately selected patients. The question arises as to whether the risk of adding another medication is less than the 
risk of washout, a procedure which may also have the advantages of providing valuable information about which current medications are actually beneficial and about the patient's true baseline condition. In addition, modern psychiatry may rely too highly on medication as the dominant answer. We may need to be more humble about our medication use and not discount the risks to which we expose our patients. While washout is accomplished differently by different providers, for the purposes of this paper, the authors taper medication allowing 5-7 half-lives as the rule of thumb for clearance. The rate of taper depends on the medication, the dose, and the amount of time the patient has been on the medicine. Guidance for tapering is presented in Figure 1.

Perhaps the most immediate crisis facing the field of psychiatry today is how to best care for the increasing numbers of patients who present with significant treatment resistance and symptomatology having failed one or more medication trials, often involving polypharmacy. A contributing factor may be that many patients are getting psychotropic medications from physicians not specializing in psychiatry and who are in need of better education regarding some of the complex patients they often end up treating. The National Comorbidity Survey found that by 2003 , one in ten adults had been treated with an antidepressant in the previous year, $73.6 \%$ of which were prescribed by a nonpsychiatric physician. ${ }^{15}$

Among other insights, a comparative analysis of specific antidepressant strategies in the STAR*D study showed modest efficacy rates for the medications used. ${ }^{16}$ This suggests that clinician persistence and encouraging treatment adherence may be more important than the specific choice of antidepressants. In their interpretation of the results of STAR $* D$, Gaynes et al ${ }^{16}$ felt that many primary care providers can treat depression in the real world and that a rational plan was more important than which drug was used. They defined this to include dosing, symptom and side effect monitoring, and assuring an adequate trial period. Other independent analyses of STAR*D have highlighted the disappointing outcomes from the study, particularly its low level of sustained improvement during follow-up. ${ }^{17-20}$ Unfortunately, the overly favorable interpretations of the results of STAR $* D^{16}$ have fostered further trial and error polypharmacy, frequently with no solid clinical or scientific rationale. For treatment-resistant depressed patients, this presents its own set of problems.

\section{Polypharmacy - rational or trial and error?}

Polypharmacy of difficult psychiatric patients is rapidly becoming the norm. In a study of the frequency of polypharmacy with three or more medications at hospital discharge for bipolar disorders or unipolar depression, polypharmacy increased from $3.3 \%$ of patients (1974-1979), to $9.3 \%$ (1980-1984), to $34 \%$ (1985-1989), and to 43.8\% (1990-1995). ${ }^{21}$ In another study undertaken between 1996 and 2005, psychiatrists significantly increased their use of polypharmacy such that outpatient visits resulting in two or more prescribed psychotropic drugs increased from $42.6 \%$ in 1996 to $59.8 \%$ in 2005 and psychiatric visits resulting in three or more such drugs being prescribed doubled, increasing from $16.9 \%$ to $33.2 \%{ }^{22}$ Unfortunately, this is a double-edged sword with risks to the patient. Apart from the increased danger of adverse events and excess side effects, not to mention increased cost of care, the authors noted that psychiatrists often got stuck when trying to switch patients to another drug; in other words, they became fearful of removing the existing medication by erroneously thinking that even if it is clearly not working, it may be better than nothing at all, and adding another agent might help. This causes the patient to be put on an additional medication instead of merely changing medications. ${ }^{22}$ In a recent article on borderline personality disorder, Gunderson discussed some concerns about polypharmacy, stating that without a clear benefit, medications should be stopped before adding a new drug. ${ }^{23}$

The practice of increased polypharmacy is at least partially driven by the momentum of recent trends that are pushing for remission, instead of merely response, as the desired outcome, yet current studies ${ }^{24}$ suggest that drug augmentation does not increase the likelihood of achieving this objective. Whether or not it is realistic to assume that medication is the only answer to psychiatric problems is a topic in itself. However, it is unclear how much one can trust the data on remission through polypharmacy, considering that selective publication and outcome reporting biases inflate benefits from drug augmentation in these studies. ${ }^{25}$

An important recent addition to the debate on the usefulness of polypharmacy is the recently reported Combining Medication to Enhance Depression Outcomes (CO-MED) study. ${ }^{24}$ In response to the STAR *D reports showing limited efficacy for a variety of antidepressant strategies, CO-MED was a large study funded by the National Institutes Of Health testing whether starting several antidepressants (with synergistic pharmacological effects) at the same time would be associated with increased efficacy. Six hundred and sixty-five patients with major depressive disorder were randomized to a 12-week treatment with escitalopram plus placebo, escitalopram plus buproprion sustained-release, or venlafaxine extended-release plus mirtazapine. Participants who experienced substantial benefit in the acute phase were 
The following principles were found to be helpful by the authors in minimizing risks with patients when tapering medications, while realizing variations occur and individualization of washout is important:

- $\quad$ Preparation and proper informed consent gains the patient's confidence and support. Initially it consists of the patient borrowing from the expertise of the provider recommending the washout. Proper management of expectations can make the difference between a successful taper and a potential crisis.

- Asking patients if they have ever missed any doses of their medicine and, if so, what symptoms they experienced, will help to identify potential risks of a more aggressive taper.

- Part of the preparation includes the patient understanding that there may be discomfort over the next few weeks. In fact, anticipating discontinuation symptoms from selective serotonin reuptake inhibitors of dizziness, dry mouth, insomnia, nausea, nervousness, sweating, anorexia, diarrhea, somnolence, and sensory disturbances (shock-like electrical sensations or "zaps") educates the patient as to realistic expectations.

- Depending on the reason for washout, allowing the patient to have input into the decision of taper speed helps confirm a sense of teamwork, avoiding anger if they experience symptoms. Whereas the more typical taper speed for selective serotonin reuptake inhibitors, for example, may be to halve the dose every 2 weeks, it has been our experience that when given this choice, patients almost uniformly choose a more rapid, yet still appropriate, taper in order to reduce the durations of any potential discomfort.

- Medications with long half-lives can temporarily be switched to similar drugs in the same class with shorter clearance (eg, escitalopram for fluoxetine or quetiapine for aripiprazole).

- Written taper schedules for each medication can help alleviate anxiety, especially when a patient is at home questioning their memory of your instructions. Compliance is as much an issue when tapering medications as it is remaining on them.

- If discontinuation symptoms do develop and cause a significant problem for the patient, allowing the use of natural products to help relieve symptoms of agitation may be beneficial. For example, augmenting with choline, inositol, 5-hydroxytryptophan, tyrosine, and/or whey protein isolate might ease anxiety and irritability. St John's wort has also been used successfully to ease washout.

- If the current schedule of medication withdrawal is causing too much discomfort, the patient should be instructed to return immediately to the previous dose until they can be seen again.

- Before attempting to washout a patient with significant psychiatric symptoms, gathering those people who serve as their support system to gain consensus is not only important for the patient's preparation, but also for their safety. The members of the support environment must agree to watch the patient for suicidal ideations or attempts, mood swings, or any other potentially life-threatening worsening of symptoms. In the rare event it is needed, they must also be prepared not to leave the patient alone. If there is not an adequate support system in place, the discontinuation should be delayed or abandoned.

Figure I Tips to help make washout easier.

enrolled in an additional 16-week continuation treatment. There was no significant difference between the response or remission rates observed in the three study arms in either the acute phase (first 12 weeks) or the continuation phase (weeks 12-28). However, the side effect burden was significantly higher in the two polypharmacy arms (escitalopram plus sustained-release buproprion: $10 \%$ maximum side effect burden at 12 weeks and $10 \%$ at 28 weeks, or venlafaxine 
extended-release plus mirtazapine: $15.2 \%$ at 12 weeks and $15.7 \%$ at 28 weeks) compared with the escitalopram-only $\operatorname{arm}(4.2 \%$ at 12 weeks and $5.2 \%$ at 28 weeks). This large, well designed, adequately powered study suggests that for a significant number of patients with major depression, polypharmacy adds to the side effect burden without an increase in efficacy.

A worrisome trend in modern psychopharmacology is the addition of atypical antipsychotics into the polypharmacy mix. Between 1997 and 2000, 30\% of antipsychotics were prescribed by nonpsychiatric physicians, ${ }^{26}$ and in 2005, the most common indications for antipsychotics were depression and bipolar disorders $(39.0 \%)$, followed by schizophrenia/other psychotic disorders (34.5\%), delirium/ dementia (7.4\%), attention-deficit/conduct-disruptive behavior disorders $(5.7 \%)$, anxiety disorders $(5.5 \%)$, and autism $(2.3 \%) .{ }^{27} \mathrm{~A}$ study in military veterans being treated for post-traumatic stress disorder found that antipsychotic medications were no better than placebo. ${ }^{28}$ Recent trends reflect increased prescribing of atypical antipsychotics for treatment-resistant depression and bipolar disorder, which are indications approved by the US Food and Drug Administration, but also for treating irritability in autism, which is not. A search of ClinicalTrials.gov ${ }^{29}$ found hundreds of additional trials underway for a wide variety of new atypical antipsychotic indications, including trials of quetiapine for irritable bowel syndrome, fibromyalgia, insomnia, and benzodiazepine replacement, as well as a host of additional nonpsychotic psychiatric disorders. ${ }^{30}$ Despite the proven efficacy of antipsychotic treatments in treatment-resistant depression and bipolar disorder, the question remains as to whether modest increases in efficacy justify the side effects and risks associated with this class of medications, which include severe metabolic abnormalities and increased cardiovascular risk.

A meta-analysis of 16 studies evaluating atypical antipsychotics as augmentation agents for treatment-resistant depression raises concerns about their increased usage. ${ }^{31}$ Likewise, it is well known that the use of polypharmacy in the elderly leads to more significant risk factors, including morbidity and mortality. ${ }^{32-35}$ As early as 1992, it was reported that psychotropic agents are the most commonly misused drugs in the elderly and are associated with increased illness severity, hospitalizations, number of physician visits, and number of pharmacies used. ${ }^{36}$

Outside of mood disorders, the risks of polypharmacy are well documented. A review of antipsychotic polypharmacy studies compared symptoms, functioning, and/or side effects in 30 studies from 1966 to 2007 in which antipsychotics were used as monotherapy or with other antipsychotics. ${ }^{37}$ The studies were stratified into those that recruited subjects who were clearly treatment-resistant to monotherapy and those studies where the subjects were not. In those studies where monotherapy had clearly failed, subjects demonstrated greater symptom control with fewer side effects when additional antipsychotics were added. Those studies where treatment resistance was not established revealed mostly negative results for symptomatology/functioning and increases in adverse events. One of the studies reported that when comparing polytherapy and monotherapy, the median final antipsychotic dose was 78\% higher, the median hospital stay was $55 \%$ longer, and the risk of adverse events was $56 \%$ higher with polytherapy. ${ }^{38}$ However, this was an open study. Two of the other studies were long-term (10 and 17 years) studies of subjects who had no established treatment resistance to monotherapy. ${ }^{39,40}$ These studies revealed an increased relative risk of death (2.5 and 2.46) in patients on multiple antipsychotics. This review of 30 studies concluded that antipsychotic polypharmacy was supported only if subjects were treatment-resistant to monotherapy. ${ }^{37}$ It should be noted that these last two studies do not account for illness severity.

Kingsbury ${ }^{41}$ makes the point that medications are often prescribed to treat a side effect of another agent. He refers to this as "bad polypharmacy", and notes that it can be seen when clinicians are afraid of changing the status quo by altering a medication that may be only partially effective. Often an additional medication is added to the existing cocktail in the hope of achieving a better therapeutic response, even though monotherapy might be sufficient. As the famous marketing pioneer, John Wanamaker, once proclaimed, "I know that half of my marketing dollars are wasted, I just don't know which half." ${ }^{42}$ This is not unlike psychiatrists evaluating a new patient presenting on numerous medications but not knowing which ones are unnecessary.

Another risk from polypharmacy is the increased adverse impact on patient health status. A recent study of 659 depressed patients found that their rate of cardiovascular problems increased from $8.8 \%$ to $30.7 \%$ after only six weeks of polypharmacy. ${ }^{43}$ The life-threatening potential of polypharmacy is such that between 2006 and 2009, 101 soldiers reportedly died accidentally while taking a toxic combination of psychotropic drugs,${ }^{44}$ and other soldiers in different military branches may have been similarly affected but unpublished. 


\section{Rational polypharmacy}

There are clearly times when polypharmacy is necessary, particularly when there are comorbidities requiring more than one class of medication or when monotherapy provides insufficient improvement. However, the dilemma for the psychiatrist is how to know if polypharmacy is needed, and if so, which of the overwhelming number of possible drug combinations are right for a particular patient. Research will never test all these options. Thus the psychiatrist is left with the perplexing decision of whether polypharmacy is warranted, along with which choice of medications should be used. The need to treat different functions of the brain or comorbid conditions may benefit a patient, but the current practice of trial-and-error prescribing is risky, especially when using more than one drug.

A number of authors have suggested guidelines for rational polypharmacy. Kingsbury ${ }^{2}$ starts by defining irrational polypharmacy, with obvious examples such as using several benzodiazepines or several antipsychotics at the same time. He also describes how irrational polypharmacy can occur in practice due to lack of knowing which is the offending drug, fear of changing one of the drugs, laziness, not wanting to rock the boat, misdiagnosis, and misunderstanding of pharmacology. Rational polypharmacy should be supported by validated double-blind, randomized controlled trials, but if they are lacking, it may be necessary to use mini-experiments within a clinic drawing on good pharmacologic decisions for drug selection, then collecting data and evaluating results on individual patients. Kingsbury goes on to suggest principles for rational polypharmacy, including review of clinical trials, overuse of benzodiazepines, a good working knowledge of pharmacology, and an awareness of potential pharmacodynamic and/or pharmacokinetic drug interactions.

Preskorn and Lacey ${ }^{45}$ recognized the difficulties in finding randomized controlled trials on polypharmacy, mostly due to the fact that these studies are expensive, usually done by pharmaceutical developers, and are limited to only those few coadministered drugs that do not have potential pharmacokinetic interactions. Rarely would a pharmaceutical company design a randomized controlled trial to study each possible drug combination that might be encountered in practice. Using the success in the Parkinson disease model, Preskorn suggests criteria for rational psychotropic polypharmacy, such as having a rational pharmacological basis, using evidence (if any) of effectiveness, maintaining safety (no worse than monotherapy), no drug interactions, using drugs with single and specific (not broad) mechanisms of action, an intermediate half-life for each drug, and linear pharmacokinetics.

\section{Role of washout}

Even after a detailed history and diagnostic workup, the psychiatrist is often faced with a key question when evaluating a treatment-resistant patient on polypharmacy for the first time, ie, how to distinguish medication effects and their adverse reactions from psychiatric symptoms. An overlooked tool in the psychiatric armamentarium to help answer this question is washing out a patient's medications, thereby allowing a re-examination of their baseline functioning.

Making certain that the patient even needs medication after years of prolonged use is good clinical care. Often the value of washout becomes clear in patients treated as children or adolescents before their brains have matured, while using street drugs, or in the midst of a hormonal storm. Yet many of these patients are never taken off their medications to see if they are still necessary once psychological and neurophysiologic maturity is completed.

For many psychiatrists, washout may seem like a barrier, if not a daunting experience. However, the reality of washout rarely lives up to these fears, and more importantly, washout is often clinically necessary as part of a thorough treatment regimen, especially in treatment-resistant patients whom the psychiatrist has not previously treated.

One study presented the findings from 2009 treatmentresistant patients from seven outpatient clinics who underwent a total medication washout as part of their psychiatric workup. ${ }^{46}$ Of these 2009 patients, only five patients $(0.25 \%)$ discontinued the washout process due to either rebounding of their original mood disorder or discontinuation symptoms, while an additional $15(0.75 \%)$ complained of an adverse response but continued through to the end of the washout. Most of the adverse events were related to mild or moderate discontinuation symptoms, with no mortality or serious morbidity in functioning. This low rate of washout failure is similar to that found in a study that involved total medication washout of treatment-resistant patients prior to randomization in 12 academic and outpatient psychiatric sites. ${ }^{47}$ In this study, only 21 of 351 patients (5.9\%) could not be randomized due to washout failure, and, as in the previous report, there was neither mortality nor significant morbidity for patients undergoing medication washout. Furthermore, another study found that only four of 51 highly symptomatic patients (7.8\%) could not tolerate washout of their antidepressant and/or mood stabilizing medications over a two-week period, while three patients experienced complete remission of their symptoms. ${ }^{48}$ This study reported that the other patients remained stable or improved on clinical measures, without worsening of symptoms during the two weeks of psychotropic washout. 
In terms of discontinuation due to intolerance, washout compares very favorably with polypharmacy in treatmentresistant patients (see Table 1). In steps $2-4$ of the STAR*D study, the discontinuation rate due to intolerance of drug augmentation ranged from $19.5 \%$ to $34.1 \%,{ }^{49}$ and in the meta-analysis of 16 atypical antipsychotic augmentation studies (mentioned earlier) it averaged 9.1\%. ${ }^{31}$ Therefore, based on these studies, ${ }^{31,49}$ the dropout rates due to adding medications to an existing regimen range from $9.1 \%$ to $34.1 \%$, ie, considerably larger than the dropout rates due to washing out medications. ${ }^{47,48}$ These comparisons highlight the relative risk of adverse events between polypharmacy and washout. While the time of recording side effects is different in washout studies (1-2 weeks) and augmentation studies (6-12 weeks), the essential point of comparison at the moment is when the decision to washout or add more medications to the therapeutic regimen is made. It is at that point that risk of washout is often unrealistically feared.

In many instances medication stacking (or adding medications when patients complain of additional symptoms that may be environmentally related or due to minor cycling, which time alone might rebalance, resulting in the use of unnecessary drugs), interactions and side effects can be a cause of psychiatric symptoms and, in fact, these symptoms may improve after washout to the point where no medication may be immediately necessary. ${ }^{50}$ In one paper, washout was reported as aiding in the diagnosis of an extremely complex case, further validating its importance in difficult patients. ${ }^{51}$ In this case, the patient's diagnosis was in question for many years, and clinicians could not determine if he had an antisocial personality disorder or schizophrenia. Neuroleptics were masking true paranoia and, as the author noted, florid delusional behaviors became apparent in the drug washout period. Although seldom used, washout should be an essential tool for psychiatrists to consider in their workup of treatment-resistant patients. Table $2 \mathrm{com}-$ pares washout and polypharmacy in terms of their risks and benefits when using either approach with treatment-resistant patients. Figure 1 provides some clinically useful tips on how to minimize the difficulties patients may have with tapering medications.

Table I Comparative estimates of complications of washout versus polypharmacy

\begin{tabular}{ll}
\hline Treatment modality & Estimates of intolerance \\
\hline Washout & $5.9 \%-7.8 \%$ \\
Polypharmacy & $9.1 \%-34.1 \%$ \\
\hline
\end{tabular}

\section{Washout is not for everyone}

While the above discussion focuses on the advantages of washout, tapering a patient off their current medications is not recommended for everyone. Clearly there are risks to washing out medications in a patient, including return or rebound of their medical/psychiatric problems, discontinuation symptoms, withdrawal (particularly with benzodiazepines), relapse, and fear of not feeling protected by whatever benefit the drugs may offer, to name a few. Even patients who presume their medication is not working may feel worse when their therapeutic agent is removed. Withdrawing from specific medications (like neuroleptics), as well as severity of illness, speed of withdrawal, and inpatient versus outpatient status, are all variables to be considered before washout. ${ }^{52}$ Patients with schizophrenia or bipolar disorder deserve special consideration because they may appear to be healthier on their medication, but may develop manic or psychotic symptoms if taken off their current regime. While it may be helpful to confirm their need for medication, a careful history from previous treatment providers is prudent. Clinical judgment must not be abandoned when making this decision, and careful tapering, including speed of washout and severity of illness, is essential.

\section{Discussion}

When faced with a decision about the next treatment to initiate in a complex or treatment-resistant patient, the typical next step is to augment or switch a medication. A medications washout can be a helpful and valuable treatment. However, when assessing the risks, it is hard to see the addition of another medication as anything but a lose-lose decision. If the new medicine works, the previous medications are still on board, adding to the risk of side effects, drug interactions, and medication stacking. If the new medication does not work, the practitioner is back to the initial problem, ie, prolonging suffering and exposing the patient to the risks of two new drugs (the one that did not work and the one about to be added). In addition, there is a risk related to delayed improvement. Washout, on the other hand, offers the opportunity for clarity, ultimately using fewer drugs by knowing which ones are necessary, decreasing the risk of drug interactions, and establishing a true baseline for the patient. It is possible that, as a result of washout, the clinician will still suggest a regimen of multiple medications (ie, polypharmacy) as the best next course of action. However, washout provides the opportunity to avoid use of unnecessary medications with their associated risks. 
Table 2 A comparison of the advantages and risks from using washout versus polypharmacy with treatment-resistant patients

\begin{tabular}{|c|c|c|}
\hline & Washout & Polypharmacy \\
\hline Advantages & $\begin{array}{l}\text { - Aids in more accurate diagnostic assessment } \\
\text { - Reveals potential negative effects and side effects from } \\
\text { current medications } \\
\text { - Reveals baseline functioning without medications that may be } \\
\text { better than current functioning } \\
\text { - May lead to reduction in polypharmacy } \\
\text { - Confidence for patient and prescriber in medication regimen, } \\
\text { helping to gain trust in the relationship } \\
\text { - Provides patients with a more realistic understanding of role } \\
\text { of their medications } \\
\text { - Helps patient compliance with long-term medications, if needed } \\
\text { - Patients feel understood and empowered } \\
\text { - Re-establishes efficacy of medications and minimizes self-blame } \\
\text { - Opportunity to provide new more effective medication regimen }\end{array}$ & $\begin{array}{l}\text { - May help with patients with combined psychiatric problems } \\
\text { - Combinations can prove beneficial for a particular indication } \\
\text { (in the case of "rational polypharmacy") } \\
\text { - Greater potential effectiveness } \\
\text { - Coverage for different phases of disorder } \\
\text { (eg, bipolar disorder) } \\
\text { - Treatment of medication side effects }\end{array}$ \\
\hline Risks & $\begin{array}{l}\text { - Risk of rebound, discontinuation, and/or withdrawal symptoms } \\
\text { - Unknown relative risk of adverse events or deterioration from } \\
\text { washout versus continued polypharmacy }\end{array}$ & $\begin{array}{l}\text { - Increased risk of adverse events, medication stacking, } \\
\text { drug-drug interactions, and causing psychiatric symptoms } \\
\text { - Masks pathophysiology, thereby making diagnosis } \\
\text { and treatment more difficult } \\
\text { - Use of potentially unnecessary and potentially } \\
\text { expensive medications }\end{array}$ \\
\hline
\end{tabular}

We want to emphasize that in our arguments we conceptualize washout as a relatively brief, time-limited period off medications which would allow more precise diagnostic and treatment interventions. We do not advocate interruption of effective treatments. For most psychiatric disorders, treatment nonadherence (ie, interruption of potentially useful treatment) is a major cause of poor treatment outcomes. For example, in schizophrenia, nonadherence with prescribed antipsychotics is associated with poor functional outcomes, ${ }^{53}$ higher risk of relapse and hospitalization, ${ }^{54}$ and increased risk of suicide. ${ }^{55}$ In our opinion, washout followed by improved assessment and treatment is actually a factor increasing adherence with long-term treatment by minimizing the burden of unnecessary medications and their associated side effects.

\section{Conclusion}

Washout may be an effective tool for physicians when the potential risks of adding further medications to a regimen outweigh the potential benefits. Re-examining the medication washout and polypharmacy challenge, the following inferences seem reasonable:

- Unwarranted fears, resistance, time constraints, or satisfaction with the "status quo" are natural hesitations that may not always be in the patient's best interests

- The most significant decision point is when a physician is trying to decide whether to washout or add more medications to the psychotropic regimen
- A potential risk of liability might exist if a physician does not demonstrate that all options (such as washout) were considered. "If a simple test is available that can confirm what is really wrong with a seriously ill patient, a doctor should not skip the test just because he's fairly sure he already knows what is wrong". ${ }^{56}$ It is incumbent upon a prescriber to consider all available information while concomitantly assessing risk

- Washing out medications in patients can be good clinical care and is done too infrequently

- A careful taper off medication with appropriate monitoring has a low degree of risk for patients being able to go off their drugs medically

- Polypharmacy can be harmful, due to side effects, drugdrug interactions, costs, and potential unnecessary stacking of medications

- When polypharmacy is appropriate, there is a paucity of literature regarding which medication options to use in which patient.

\section{Disclosure}

DAH is an employee of CNS Response Inc, holds stock in the company, and has also received speaking honoraria from Wyeth, Forest Laboratories, Cephalon, Eli Lilly, Pfizer, and GlaxoSmithKline, but not within the last 5 years. MS has served as consultant to CNS Response Inc, and holds stock in the company. JMG is a consultant to, and holds shares in, CNS Response Inc. Prior to the last two years 
DVI has received research support from Aspect Medical Systems, Forest Laboratories, Janssen Pharmaceutica, and has received speaker honoraria from Eli Lilly and Co, Forest Laboratories, and Pfizer Inc. In the past two years, DVI has received research support from Euthymics Bioscience Inc, Forest Laboratories, and NeoSync Inc, speaker honoraria from Reed-Elsevier Medical, and has been a consultant to CNS Response Inc.

\section{References}

1. NASMHPD Medical Directors' Technical Report on Psychiatric Polypharmacy. Sep 2001. National Association of State Mental Health Program Directors Medical Directors Council and State Medicaid Directors. Available from: http://www.nasmhpd.org/general_ files/publications/med_directors_pubs/Polypharmacy. Accessed Septemeber 18, 2011.

2. Kingsbury SJ, Yi D, Simpson GM. Psychopharmacology: rational and irrational polypharmacy. Psychiatr Serv. 2001;52(8):1033-1036.

3. Texas Department of Aging and Disability Services. Polypharmacy definition developed by DADS Pharmacy Services, May 2009. Available from: http://www.dads.state.tx.us/qualitymatters/qcp/polypharmacy. Accessed August 14, 2011.

4. Stroup TS, McEvoy JP, Swartz MS, et al. The National Institute of Mental Health Clinical Antipsychotic Trials of Intervention Effectiveness (CATIE) project: schizophrenia trial design and protocol development. Schizophr Bull. 2003;29(1):15-31.

5. Rush AJ, Fava M, Wisniewski SR, et al; STAR*D Investigators Group. Sequenced treatment alternatives to relieve depression $\left(\mathrm{STAR}^{*} \mathrm{D}\right)$ : rationale and design. Control Clin Trials. 2004;25(1):119-142.

6. Goldberg JF, Brooks JO 3rd, Kurita K, et al. Depressive illness burden associated with complex polypharmacy in patients with bipolar disorder: findings from the STEP-BD. J Clin Psychiatry. 2009;70(2):155-162.

7. DePaulo JR. Bipolar disorder treatment: an evidence-based reality check. Am J Psychiatry. 2006;163(2):175-176.

8. Watanabe N, Omori IM, Nakagawa A, et al. Safety reporting and adverse-event profile of mirtazapine described in randomized controlled trials in comparison with other classes of antidepressants in the acute-phase treatment of adults with depression: systematic review and meta-analysis. CNS Drugs. 2010;24(1):35-53.

9. Cipriani A, La Ferla T, Furukawa TA, et al. Sertraline versus other antidepressive agents for depression. Cochrane Database Syst Rev. 2009;15(2):CD006117.

10. Nakagawa A, Watanabe N, Omori IM, et al. Efficacy and tolerability of milnacipran in the treatment of major depression in comparison with other antidepressants: a systematic review and meta-analysis. CNS Drugs. 2008;22(7):587-602.

11. Cipriani A, Geddes JR, Furukawa TA, Barbui C. Metareview on shortterm effectiveness and safety of antidepressants for depression: an evidence-based approach to inform clinical practice. Can J Psychiatry. 2007;52(9):553-562.

12. Insel TR. Disruptive insights in psychiatry: transforming a clinical discipline. J Clin Invest. 2008;119(4):700-705.

13. Woods SW, Morgenstern H, Saksa JR, et al. Incidence of tardive dyskinesia with atypical versus conventional antipsychotic medications: a prospective cohort study. J Clin Psychiatry. 2010;71(4): 463-474.

14. Greenberg G. Inside the battle to define mental illness. Wired. Jan 2011.

15. Mojtabai R, Olfson M. National patterns in antidepressant treatment by psychiatrists and general medical providers: results from the national comorbidity survey replication. J Clin Psychiatry. 2008;69(7): 1064-1074.

16. Gaynes B, Rush A, Trivedi M, et al. The STAR*D study: Treating depression in the real world. Cleve Clin J Med. 2008;75(1):57-66.
17. Fava GA, Tomba E, Grandi S. The road to recovery from depression don't drive today with yesterday's map. Psychother Psychosom. 2007; 76(5):260-265.

18. Ghaemi SN. Why antidepressants are not antidepressants: STEP-BD, STAR*D, and the return of neurotic depression. Bipolar Disord. 2008;10(3):957-968.

19. Pigott HE, Leventhal AM, Alter GS, Boren JJ. Efficacy and effectiveness of antidepressants: current status of research. Psychother Psychosom. 2010;79(5):267-279.

20. Pigott HE. STAR*D: A tale and trail of bias. Ethical Hum Psychol Psychiatry. 2011;13(1):6-28.

21. Frye MA, Ketter TA, Leverich GS, et al. The increasing use of polypharmacotherapy for refractory mood disorders: 22 years of study. J Clin Psychiatry. 2000;61(1):9-15.

22. Mojtabai R, Olfson M. National trends in psychotropic medication polypharmacy in office-based psychiatry. Arch Gen Psychiatry. 2010; 67(1):26-36.

23. Gunderson JG. Clinical practice. Borderline personality disorder. N Engl J Med. 2011;364(21):2037-2042.

24. Rush AJ, Trivedi MH, Stewart JW, et al. Combining Medications to Enhance Depression Outcomes (CO-MED): acute and long-term outcomes of a single-blind randomized study. Am J Psychiatry. 2011; 168(7):689-701.

25. Yury CA, Fisher JE, Antonuccio DO, Valenstein M, Matuszak J. Meta-analysis of antidepressant augmentation: piling on in the absence of evidence. Ethical Hum Psychol Psychiatry. 2009;11(3):171-182.

26. Van Brunt DL, Gibson PJ, Ramsey JL, Obenchain R. Outpatient use of major antipsychotic drugs in ambulatory care settings in the United States.1997-2000. Med Gen Med. 2003;5(3):16.

27. Mark, Tami L. For what diagnoses are psychotropic medications being prescribed? A nationally representative survey of physicians. CNS Drugs. 2010;24(4):319-326.

28. Krystal JH, Rosenheck RA, Cramer JA, et al. Adjunctive risperidone treatment for antidepressant-resistant symptoms of chronic military service-related PTSD: a randomized trial. JAMA. 2011;306(5): 493-502.

29. ClinicalTrials.gov. A review of clinical trials on the website. Available from http://www.clinicaltrials.gov/ct2/results?term=atypical+antipsyc hotics/. Accessed October 10, 2011. Accessed March 11, 2011.

30. Nardo JM. The gird. One Boring Old Man Blog. Available from: http://1boringoldman.com/index.php/2011/01/04/the-grid/. Accessed February 18, 2011.

31. Nelson CJ, Papakostas GI. Atypical antipsychotic augmentation in major depressive disorder: a meta-analysis of placebo-controlled randomized trials. Am J Psychiatry. 2009;166(9):980-991.

32. Hajjar ER, Cafiero AC, Hanlon JT. Polypharmacy in elderly patients. Am J Geriatr Pharmacother. 2007;5(4):345-351.

33. Gurvich T, Cunningham JA. Appropriate use of psychotropic drugs in nursing homes. Am Fam Physician. 2000;61(5):1437-1446.

34. Feil D, Weinreb J, Sultzer D. Psychiatric disorders and psychotropic medication use in elderly persons with diabetes. Ann Longterm Care. 2006;14(7):39-47.

35. Nicole Damestoy N, Collin J, Lalande R. Prescribing psychotropic medication for elderly patients: some physicians' perspectives. CMAJ. 1999;161(2):143-145.

36. Montamat SC, Cusack B. Overcoming problems with polypharmacy and drug misuse in the elderly. Clin Geriatr Med. 1992;8(1):143-158.

37. Goren JL, Parks JJ, Ghinassi FA, et al. When is antipsychotic polypharmacy supported by research evidence? Implications for QI. Jt Comm J Qual Patient Saf. 2008;34(10):571-582.

38. Centorrino F, Goren JL, Hennen J, Salvatore P, Kelleher JP, Baldessarini RJ. Multiple vs single antipsychotic agents for hospitalized psychiatric patients: Case-control study of risks versus benefits. Am J Psychiatry. 2004;161(4):700-706.

39. Joukamaa M, Heliovaara M, Knekt P, Aromaa A, Raitasalo R, Lehtinen V. Schizophrenia, neuroleptic medication and mortality. Br J Psychiatry. 2006;188:122-127. 
40. Waddington JL, Youssef HA, Kinsella A. Mortality in schizophrenia: Antipsychotic polypharmacy and absence of adjunctive anticholinergics over the course of a 10-year prospective study. Br J Psychiatry. 1998;173:325-329.

41. Kingsbury L. Psychiatric polypharmacy: the good, the bad, and the ugly. Psychiatr Times. 2007;24(4):1-3.

42. John Wanamaker. US department store merchant1838-1922. Available from: http://en.wikipedia.org/wiki/John_Wanamaker. Accessed April 14, 2011.

43. Helwick C. Polypharmacy is common in psychiatry, but is more better? Medscape Medical News. Available from: http://www.medscape.com/ viewarticle/728132. Accessed

44. Dao J, Carey B, Frosh D. For some troops, powerful drug cocktails have deadly results. New York Times. February 12, 2011. Available from: http:// www.nytimes.com/2011/02/13/us/13drugs.html?ref=benedictcarey. Accessed September 16, 2011.

45. Preskorn SH, Lacey RL. Polypharmacy: when is it rational? J Psychiatr Pract. 2007;13(2):97-105.

46. Hoffman DA, DeBattista C, Schiller M. Medication washout: imposing discontinuation or a diagnostic and therapeutic tool? Poster 230 presented at the US Psychiatric and Mental Health Congress, San Diego, CA, October 30-November 2, 2008.

47. DeBattista C, Kinrys G, Hoffman D, et al. The use of referenced-EEG (rEEG) in assisting medication selection for the treatment of depression. J Psychiatr Res. 2011;45(1):64-75.

48. Grunebaum MF, Oquendo MA, Burke AK, et al. Clinical impact of a 2 week psychotropic medication washout in unipolar depression inpatients. J Affect Disord. 2003;75(3):291-296.
49. Rush AJ, Trivedi MH, Wisniewski SR, et al. Acute and longer-term outcomes in depressed outpatients requiring one or several treatment steps: a STAR*D report. Am J Psychiatry. 2006;163(11):1905-1917.

50. Hoffman DA. First, do no harm - a basic tenet in jeopardy? J Neurother. 2007;10(4):53-61.

51. Patel JK, Green AI, Kalinowski A, Tsuang MT. Evaluation of a complex case: the value of a drug washout period. Am J Psychiatry. 1997; 154(12):1747-1750.

52. Viguera AC, Baldessarini RJ, Hegerty JD, van Kammen DP, Tohen M. Clinical risk following abrupt and gradual withdrawal of maintenance neuroleptic treatment. Arch Gen Psychiatry. 1997;54(1):49-55.

53. Ascher-Svanum H, Faries DE, Zhu B, Ernst FR, Swartz MS, Swanson JW. Medication adherence and long-term functional outcomes in the treatment of schizophrenia in usual care, J Clin Psychiatry. 2006; 67(3):453-460.

54. Novick D, Haro JM, Suarze D, Perez V, Dittmann RW, Haddad PM. Predictors and clinical consequences of non-adherence with antipsychotic medication in the outpatient treatment of schizophrenia. Psychiatry Res. 2010;176(2-3):109-113.

55. Dutta R, Murray RM, Allardyce J, Jones PB, Boydell J. Early risk factors for suicide in an epidemiological first episode psychosis cohort. Schizophr Res. 2011;126(1-3):11-19.

56. Friedman R, Malone P. Rules of the Road: A Plaintiff Lawyer's Guide to Proving Liability. 2nd ed. Portland, OR: Trial Guides LLC; 2010.
Neuropsychiatric Disease and Treatment

\section{Publish your work in this journal}

Neuropsychiatric Disease and Treatment is an international, peerreviewed journal of clinical therapeutics and pharmacology focusing on concise rapid reporting of clinical or pre-clinical studies on a range of neuropsychiatric and neurological disorders. This journal is indexed on PubMed Central, the 'PsycINFO' database and CAS, and is the official

\section{Dovepress}

journal of The International Neuropsychiatric Association (INA). The manuscript management system is completely online and includes a very quick and fair peer-review system, which is all easy to use. Visit http://www.dovepress.com/testimonials.php to read real quotes from published authors. 